\title{
EXPANSIÓN LINEAL Y PUNTO DE SATURACIÓN DE LAS FIBRAS DE LA Guadua angustifolia Kunth
}

\section{Linear expansion and fiber saturation point of Guadua angustifolia Kunth}

\author{
Mateo Gutiérrez González ${ }^{1}$, Jessika Isabel Bonilla Santos², María Fernanda Cruz Amado ${ }^{3}$ \\ \& Juan Guillermo Quintero Aranzalez ${ }^{4}$
}

Gutiérrez-González, M., Bonilla-Santos, J.I., Cruz-Amado, M.F., \& Quintero-Aranzalez, J.G. (2018). Expansión lineal y punto de saturación de las fibras de la Guadua angustifolia Kunth. Colombia Forestal, 21(1), 69-80

Recepción: 13 de marzo de 2017

\section{Resumen}

La Guadua angustifolia Kunth es una especie de bambú que ha sido ampliamente utilizado en la construcción. Gracias a su capacidad de absorber agua, la G. angustifolia experimenta cambios en sus dimensiones, resistencia y rigidez debido a la variación del contenido de humedad. El punto de saturación de las fibras (PSF) se alcanza cuando a un determinado contenido de humedad el material no presenta cambios dimensionales ni variaciones en la resistencia mecánica. Para determinar el PSF de G. angustifolia se analizaron probetas de la parte inferior, intermedia y superior de la guadua. Se utilizaron dos metodologías: el primer método midió la variación dimensional, encontrando que el PSF está alrededor de $34.05 \%$; el segundo determinó la variación de la resistencia a la compresión paralela, encontrando que el PSF está alrededor de $34 \%$. Por último, se presentaron los factores de modificación de la resistencia a compresión para diferentes contenidos de humedad, así como la ecuación de expansión lineal del material.

Palabras clave: contenido de humedad, higroscópico, punto de saturación de las fibras, resistencia a la compresión, rigidez, variación dimensional.
Aceptación: 28 de agosto de 2017

\begin{abstract}
Guadua angustifolia Kunth is a bamboo species, which has been widely used in construction. G. angustifolia is considered a hygroscopic material due to its capacity of absorb water, leading to changes in dimensions, strength, and stiffness due to variation of moisture content. The fibre saturation point (FSP) is reached when at certain moisture content, the material does not present dimensional changes and does not undergo variations in the mechanical strength. Specimens from the upper, middle and upper part of the bamboo were analysed in order to determine the FSP. Two methods were used to determine the FSP; the first method used the dimensional variation of the material at different moisture contents. The results showed that FSP is around 34.05\%. The second method allowed verifying the FSP of Guadua angustifolia Kunth from the analysis of the parallel compression strength at different moisture contents. This method found that FSP was around 34\%. Finally, a modification factor due to moisture content for compression strength was presented, as well as the equation for linear expansion of the material.
\end{abstract}

Key words: moisture content, hygroscopic, fibre saturation point, compression strength, stiffness, dimensional variation.

1 School of Civil Engineering, The University of Queensland. Brisbane, Australia.m.gutierrezgonzalez@uq.edu.au. Autor para correspondencia.

2 Facultad de Ingeniería, Universidad La. Bogotá D.C, Colombia. jessikaisabel.bonilla@ulagrancolombia

3 Facultad de Ingeniería, Universidad La Gran Colombia. Bogotá D.C, Colombia. mariafernando.cruz@ulagrancolombia.edu.co

4 Facultad de Ingeniería, Universidad La Gran Colombia. Bogotá D.C, Colombia. juanguillermo.quintero@ulagrancolombia.edu.co 


\section{INTRODUCCIÓN}

La G. angustifolia Kunth es una especie de bambú que se ha sido usado por diferentes comunidades de América latina desde hace cientos de años. Durante las últimas décadas, el interés por utilizar este material para aplicaciones en la industria de muebles, fabricación de utensilios, artesanías y construcción ha crecido considerablemente. Su uso se ha dado gracias a los conocimientos y técnicas aprendidos a través de la experiencia, pero poco ha sido el desarrollo ingenieril del material, lo que ha conducido en muchos casos a un uso inadecuado o incorrecto (Correal, 2016). Particularmente, la industria de la construcción ha utilizado la G. angustifolia para la elaboración de viviendas o puentes, los cuales han sido construidos en su mayoría sin ningún tipo de diseño estructural y con baja supervisión técnica (Dowling, 2004; Luna et al, 2011), conduciendo a errores que han Ilevado al colapso de estructuras. Desde comienzos del nuevo milenio, Colombia ha liderado la elaboración de normas que han permitido entender muchas de las características de la guadua, con el fin de que las industrias que utilizan el material puedan incrementar su uso y mejorar su comportamiento, para así poder darle un mejor y seguro aprovechamiento (Gatóo et al., 2014).

La G. angustifolia es un material higroscópico que puede absorber agua del ambiente que le rodea. Las variaciones en el contenido de humedad dentro de ciertos rangos permiten que el material experimente cambios tanto en su peso como en sus dimensiones, debido a que el agua puede depositarse tanto en las cavidades como en las paredes celulares de la planta. El contenido de humedad en el bambú varía significativamente dependiendo del estado en el que se encuentre. Un bambú joven recién cortado, en condición verde, puede tener contenidos de humedad de hasta un $150 \%$ (Hidalgo, 2003; Liesse \& Köhl, 2015). Después de sufrir un proceso de secado natural o controlado, el bambú pierde humedad hasta equilibrarse con el ambiente en el que se encuentra. A este contenido de humedad se le conoce como el contenido de humedad de equilibrio, y según investigaciones realizadas anteriormente este contenido puede estar entre un $6 \%$ y un $30 \%$ (Hamdan et al., 2007; Gutiérrez \& Briceño, 2015). Para describir la manera en la que se deposita el agua, se puede decir que una planta de bambú que se encuentra húmeda puede experimentar un proceso de secado. En principio, la primera cantidad de agua en secarse es la que se encuentra en las cavidades celulares y se conoce como agua libre, posteriormente se inicia la pérdida del agua que se encuentra saturando las paredes celulares, la cual se conoce como agua higroscópica (Cartagena, 1984). Cuando un material como la madera o el bambú tiene totalmente saturadas de agua las paredes celulares, a ese valor de contenido de humedad se le conoce técnicamente como el punto de saturación de las fibras (PSF) (Liesse \& Köhl, 2015) y se caracteriza por que el material no presenta variaciones dimensionales ni variaciones en sus propiedades mecánicas para contenidos de humedad por encima de ese punto (Gutiérrez, 2011). En el bambú, el PSF está influenciado por la composición del tejido y la cantidad de constituyentes químicos. Algunos autores (Sharma 1988; Hamdan et al. 2007) han indicado que, en promedio, el PSF del bambú esta alrededor del 17-25\%. Sin embargo, estos valores relativamente bajos fueron obtenidos a partir de metodologías en donde se sometieron muestras a ambientes con $100 \%$ de humedad relativa en la atmosfera, lo cual no representa las condiciones de saturación reales de una muestra (Liesse \& Köhl, 2015).

En el año 2010 se actualizó el reglamento colombiano de construcción sismo resistente NSR10, y específicamente en el Título G "Estructuras de Madera y Guadua" (AIS, 2010), se consignaron diferentes valores correspondientes a las propiedades físicas y mecánicas de la guadua, los cuales son necesarios para realizar el diseño estructural de una edificación construida con guadua como elemento principal. En el reglamento se incluyeron factores de modificación de la resistencia dependiendo el 
contenido de humedad y se establecieron rangos de contenido de humedad entre los que se presentaba variación de las propiedades mecánicas. Sin embargo, el valor específico para el PSF de la G. angustifolia no fue mencionado. Para cualquier material natural e higroscópico de uso estructural, el PSF permite determinar los intervalos de contenido de humedad entre los que pueden variar tanto la resistencia como las dimensiones geométricas del material. Además, permite calcular los factores de modificación por contenido de humedad, utilizados en la modelación de estructuras de guadua, los cuales se encuentran establecidos en la NSR-10 en la tabla G.12.7.5 "Coeficientes de modificación por contenido de humedad $\left(C_{m}\right)^{\prime \prime}$. Estos factores muestran que, a medida que aumenta el contenido de humedad, la rigidez y la resistencia del material disminuyen (AIS, 2010).

De acuerdo con diversos investigadores (AIS, 2010; Gutiérrez, 2011; Jiang, 2012; Wang, 2013; Xu, et al 2014; Kamiski et al, 2016b), la variación en el contenido de humedad es uno de los factores de mayor afectación en las propiedades mecánicas del bambú. Algunos autores han encontrado que el bambú presenta disminuciones de resistencia de hasta un $30 \%$ cuando se alcanza el PSF (Jiang et al, 2012; Xu, et al 2014). Esta pérdida de resistencia es notoria en solicitaciones de carga a compresión paralela a la fibra y cortante paralelo; a pesar de ello, otros estudios también han demostrado que ante solicitaciones de tensión paralela la resistencia mecánica es prácticamente indiferente al aumento del contenido de humedad debido a que solo se observan disminuciones de un 5 \% (Gutiérrez \& Takeuchi, 2014). De estos estudios también se ha encontrado que la resistencia se vuelve constante cuando el contenido de humedad varía por encima del PSF.

La construcción con G. angustifolia ha estado en constante crecimiento durante los últimos años, sus excelentes propiedades mecánicas han permitido la construcción de elementos estructurales con características similares de otros materiales convencionales como el concreto o el acero. La industria de la construcción ha volcado su interés a utilizar materiales más amigables con el ambiente, altamente renovables, que tengan bajos costos energéticos y económicos, y que ofrezcan una alternativa estéticamente atractiva, aspectos que las construcciones en guadua cumplen a cabalidad (Zea, 2015). Debido al excelente desempeño que ha tenido en diferentes eventos sísmicos (Kamiski et al, 2016a), y gracias a la alta relación de resistencia-peso, el reglamento colombiano de construcciones sismo resistentes (AIS, 2010) ha permitido la construcción de viviendas con G. angustifolia como material estructural principal. Todas estas razones le permiten a este material convertirse en una solución técnica y económica ante la problemática social que implica el déficit de vivienda y el alto costo que esto representa en muchos países tropicales y latinoamericanos que cuentan con este recurso natural. Sin embargo, la industria de la construcción en G. angustifolia todavía enfrenta grandes desafíos. Es necesario determinar muchas de las variables que afectan su comportamiento físico y mecánico, como lo es el contenido de humedad. También debe entenderse el comportamiento mecánico de las estructuras, las teorías de análisis estructural que puedan aplicarse a materiales ortotrópicos, el diseño y comportamiento de las conexiones y la resistencia frente al fuego, con el fin de poder sacar un mejor provecho de este material. Esta investigación plantea dos procedimientos que permitieron encontrar el PSF de la G. angustifolia, así como establecer el rango de contenido de humedad y la manera en el que varía tanto la resistencia mecánica a la compresión paralela como las dimensiones geométricas del material.

\section{MATERIALES Y MÉTODOS}

\section{Materiales}

Para la realización de los ensayos se utilizó la $G$. angustifolia Kunth, extraída del municipio de Calarcá, Quindío, con edad promedio de cuatro años 
que cumpliera con las especificaciones de calidad especificadas en la NTC 5301 (Icontec, 2007b). Para los ensayos se tomaron probetas de diferentes partes la guadua en altura (inferior o cepa, intermedia o basa y superior o sobrebasa). Se escogió una muestra de 30 probetas con nudo y 30 probetas sin nudo. Cada probeta tuvo las siguientes dimensiones aproximadamente: $10 \mathrm{~cm}$ de alto, 3.5 cm de ancho y el espesor correspondía al espesor del culmo del cual fue extraída la probeta.

\section{Metodología}

\section{Identificación del método para variar el} contenido de humedad al interior del material Las probetas se secaron en el horno durante 24 horas a una temperatura de $100 \pm 3^{\circ} \mathrm{C}$, con el fin de obtener probetas con un $0 \%$ de humedad. Cuando las probetas ya estaban secas, se sometieron a una variación del contenido de humedad para poder establecer el rango en el debían hacerse las mediciones de variación dimensional. Se sumergió cada grupo de probetas en agua en un intervalo de tiempo fijo con el objetivo de determinar el contenido de humedad en el cual las dimensiones de cada una de las probetas permanecieran constantes. El proceso de inmersión de las probetas a diferentes tiempos ya había sido utilizado previamente en otras investigaciones (Gutiérrez, 2011; Xu et al, 2014). Igualmente, se escogió esta metodología pues era posible obtener contenidos de humedad mayores al $30 \%$ en menos de tres horas. Otras metodologías reportadas en la literatura (Hamdan et al, 2007), utilizaron cámaras de humedad relativa controlada; sin embargo, este procedimiento no permite alcanzar contenidos de humedad en el material mayores al $26 \%$, lo cual limita significativamente el alcance de la investigación.

\section{Curvas de variación dimensional}

Para determinar la variación dimensional a partir del contenido de humedad del material se estudiaron grupos de 30 probetas con y sin nudo, estudiando las tres principales partes de la planta: cepa, basa y sobrebasa, esto con el fin de tener en cuenta la variación en la densidad de fibras en el material (Grosser \& Liese, 1971), para un total de 180 probetas de estudio. Las probetas fueron marcadas de acuerdo con su procedencia y características, así como también se marcó las dimensiones geométricas a medir según cada condición diferente de humedad. A cada una de las probetas se le midieron sus tres dimensiones (longitudinal, tangencial y radial) mediante un calibrador pie de rey digital vernier para así obtener un valor promedio de cada una de ellas. Las probetas se sumergieron durante diferentes tiempos previamente definidos y en cada uno se tomaron las dimensiones en todas las direcciones, así como el peso de la probeta, para así determinar posteriormente el $\mathrm{CH}$ al que se realizó cada medición.

La determinación del contenido de humedad se llevó a cabo según lo mencionado en la Norma Técnica Colombiana NTC 5525 "Métodos de ensayo para determinar las propiedades físicas y mecánicas de la Guadua angustifolia Kunth" (Icontec, 2007a). El contenido de humedad se calculó mediante la siguiente expresión:

de humedad

$C H(\%)=\frac{W_{i}-W_{0}}{W_{0}} * 100$ ecuación 1. Contenido

Dónde $\mathrm{CH}(\%)$ corresponde al contenido de humedad expresado en porcentaje, $W_{i}$ es el peso inicial de la probeta y $W_{0}$ es el peso seco o anhidro de la probeta.

Con los datos obtenidos, se calculó el porcentaje de variación dimensional de cada una de las probetas, teniendo en cuanta cada una de las variables mencionadas anteriormente, con el fin de observar la variación en la longitud de cada dimensión estudiada (longitudinal, tangencial y radial), así como también el porcentaje de contenido de humedad. Se realizó un análisis estadístico mediante el criterio de chauvenet para eliminar datos atípicos para cada porcentaje de expansión lineal en función del contenido de humedad, en cada una de las partes de la guadua. Con estos datos se realizaron las gráficas de expansión dimensional 
(\%) versus contenido de humedad (\%). Se estableció que el PSF correspondería al contenido de humedad a partir del cual las probetas no presentaran un aumento porcentual en sus dimensiones.

\section{Curvas de resistencia a la compresión paralela} a las fibras a partir de diferentes contenidos de humedad

Para la obtención de la curva de resistencia a compresión vs. contenido de humedad, se utilizaron un total de 75 probetas pertenecientes a las diferentes partes del culmo (cepa, basa y sobrebasa). En las probetas utilizadas se midió el diámetro externo e interno para determinar el área y con este valor poder hallar el esfuerzo que soporta cada probeta.

Para variar el contenido de humedad, las probetas fueron sumergidas en agua durante los tiempos encontrados previamente en los ensayos realizados para variar las dimensiones de las probetas. Se pesaron las probetas húmedas y se llevaron a la máquina de compresión Versa Tester de la Universidad La Gran Colombia, siendo ensayadas con los procedimientos descritos en la NTC 5525 (González \& Díaz, 1992; Icontec, 2007a; González \& Leguizamón, 2012).

Con las muestras ya ensayadas, era necesario conocer el contenido de humedad, por esta razón se introdujeron en el horno para secarlas y calcular el $\mathrm{CH}$ de cada una de las probetas siguiendo el procedimiento establecido en la NTC 5525 (Icontec, 2007a). Con los datos obtenidos se realizó una gráfica de esfuerzo a compresión paralela contra contenido de humedad encontrando así el PSF de la guadua, que se presenta cuando la resistencia a la compresión permanece constante a pesar del aumento del contenido de humedad.

\section{RESULTADOS}

Al analizar los valores obtenidos para la expansión longitudinal se puede observar que en general, para la dirección longitudinal, las probetas no se expanden más de un $0.8 \%$ para contenidos de humedad de hasta el $60 \%$. Esta situación también se presentó en probetas de todas las partes de la guadua en altura (cepa, basa y sobrebasa). En este sentido, se puede decir que el bambú presenta un comportamiento similar al de la madera ya que la expansión longitudinal del material en función del contenido de humedad del material es menor al $1 \%$, lo cual es prácticamente insignificante para un elemento estructural. Debido a la poca relación entre variación longitudinal y contenido de humedad, estos resultados no fueron tenidos en cuenta para encontrar el PSF.

Para determinar el PSF se decidió analizar de manera independiente cada una de las variables involucradas, teniendo en cuenta únicamente los resultados de las dos dimensiones que registraron mayor variación dimensional (tangencial y radial). Estas variaciones dimensionales se analizaron para probetas de la cepa y basa, y para probetas con nudo y sin nudo. Se decidió no utilizar los resultados obtenidos para la sobrebasa ya que no presentaron un comportamiento similar al que presentaron las otras dos variables. Al ver que los resultados para la cepa y la basa fueron similares, se hizo el análisis sin importar la dimensión y la procedencia, así como la presencia o ausencia de nudo. Se sabe que la parte de la guadua que presenta mayor tejido parenquimático es la cepa, debido a que en su estructura interna hay una menor densidad de fibras. Los resultados encontrados evidencian que la cepa absorbe mayores contenidos de humedad en un menor tiempo, comparado con los contenidos absorbidos en la basa y sobrebasa. Esto se debe a que el tejido parenquimático es el principal responsable de la absorción de agua en el material.

En las figuras 1a y 1 b se presentan la variación dimensional tangencial y radial versus contenido de humedad para probetas de cepa y basa respectivamente. Al observar un comportamiento similar en cada una de las variables analizadas, se decidió analizar en una misma gráfica el comportamiento de la dimensión tangencial y radial en ambas 
partes, con el objetivo de determinar un único valor del PSF (figura 2). Igualmente, se expresó la expansión como una función lineal del $\mathrm{CH}$. Estas gráficas presentan un resumen detallado de los

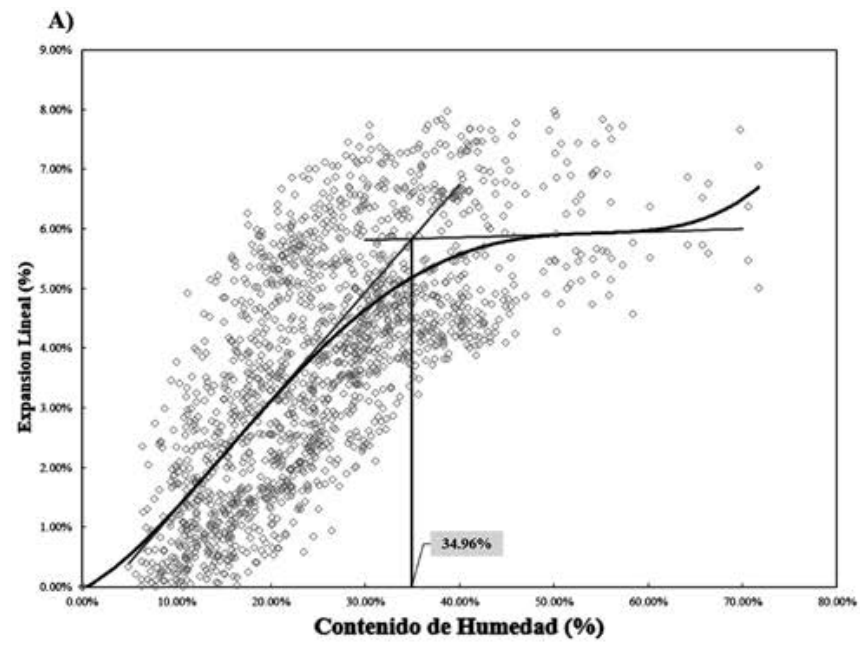

resultados obtenidos para cada una de las variables analizadas de acuerdo a la expansión lineal en la dirección tangencial y radial de la G. angustifolia Kunth (figuras 1a, 1 b y 2).

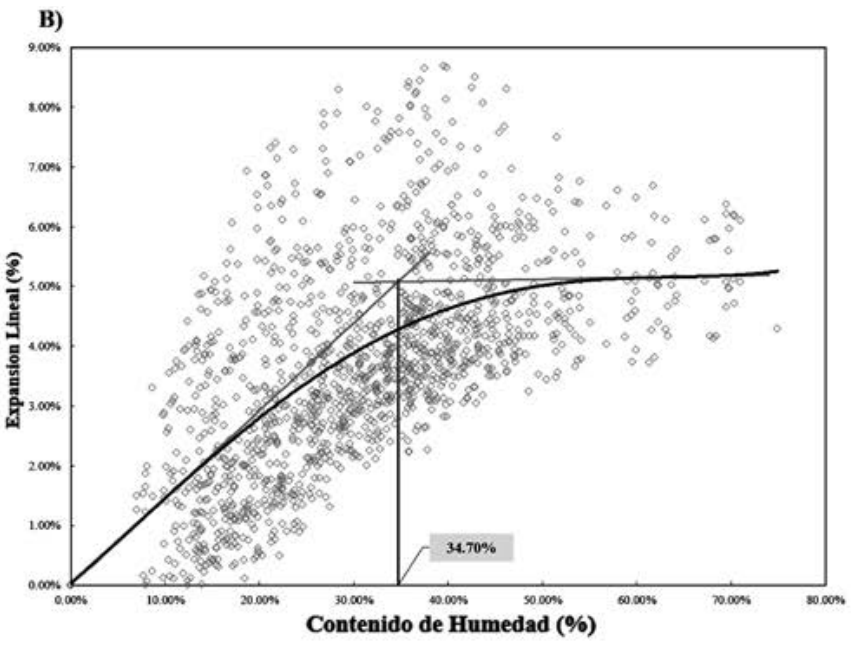

Figura 1. Expansión lineal de cepa en la dirección radial y tangencial en función del contenido de humedad (A) expansión lineal de basa en la dirección radial y tangencial en función del contenido de humedad (B).

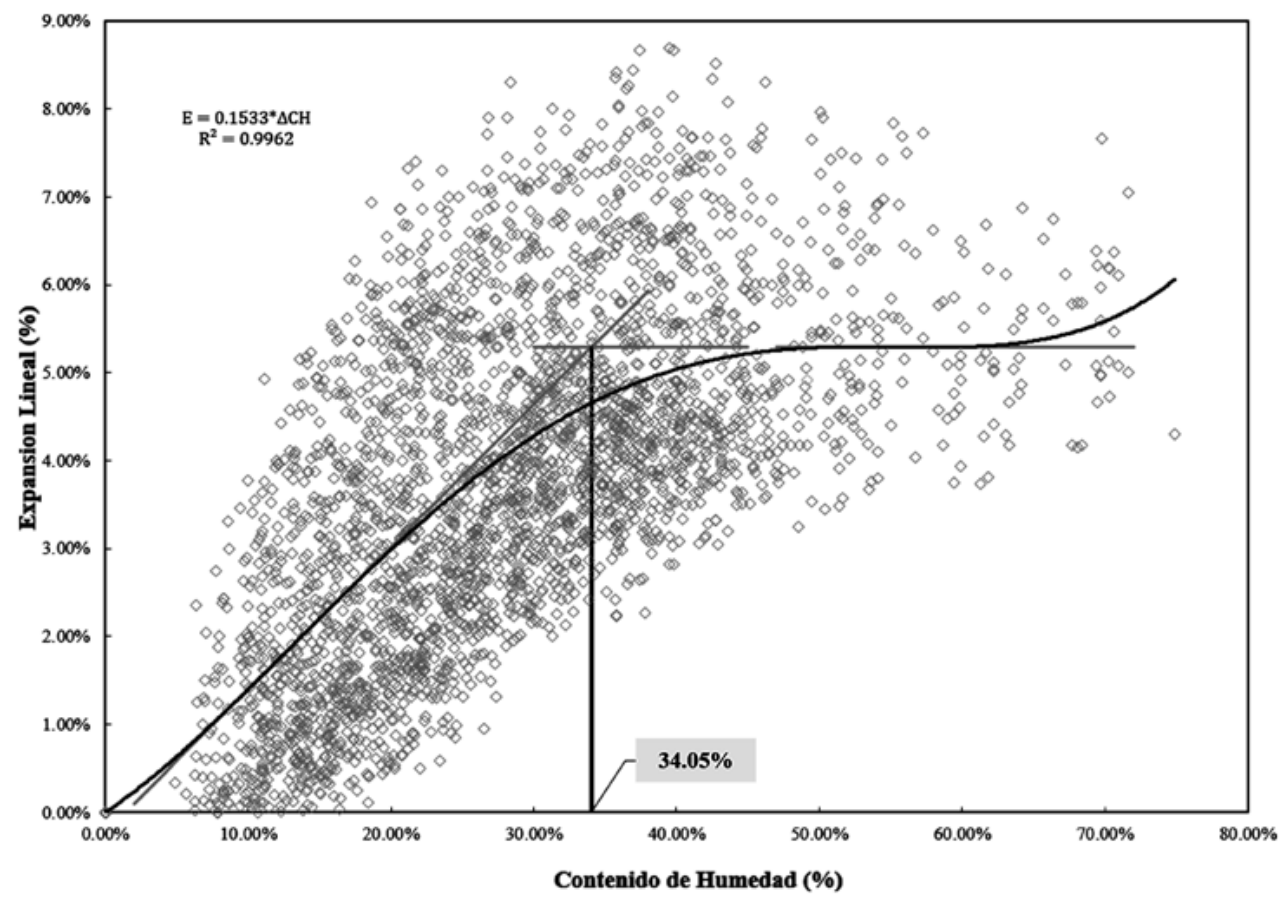

Figura 2. Expansión lineal de cepa y basa en la dirección radial y tangencial en función del contenido de humedad. 
A partir de estos resultados se utilizó la parte lineal de la figura 2, con el fin de tener una ecuación que permita calcular la expansión lineal de la guadua, cuando el material sufre un aumento en el contenido de humedad, siempre y cuando el contenido de humedad se encuentre por debajo del PSF, en este caso, $34.05 \%$. Por lo tanto, la expansión lineal que puede sufrir un elemento de guadua rolliza en su dirección radial o tangencial se puede expresar como:

$E(\%)=0.1533\left(\mathrm{CH}_{f}(\%)-\mathrm{CH}_{i}(\%)\right)$ ecuación 2. Expansión lineal de la guadua

Para $\mathrm{CH}_{f} \leq \mathrm{PSF}(\mathrm{PSF}=34 \%)$

Donde $E$ corresponde a la expansión lineal de la guadua en porcentaje, $\mathrm{CH}_{f}$ es el contenido final de la guadua, $\mathrm{CH}_{i}$ es el contenido inicial de la guadua antes del aumento en su humedad. Ambos contenidos deben expresarse en porcentaje para obtener la expansión lineal en porcentaje.
Después de analizada la variación dimensional y los respectivos resultados del PSF de la guadua, se procedido a realizar el análisis del comportamiento de la resistencia mecánica a la compresión paralela versus el contenido de humedad. Al evaluar la gráfica de resistencia vs. contenido de humedad, se espera que la resistencia a la compresión disminuya a medida que aumenta el $\mathrm{CH}$ en el material. Sin embargo, se creería que a partir del PSF la resistencia se mantenga constante. En la figura 3 se presentan los resultados de resistencia mecánica a la compresión paralela para las tres diferentes partes de la guadua en altura. Como se ha demostrado anteriormente, la presencia o ausencia de nudo en las probetas no representa diferencias significativas en los esfuerzos últimos a compresión (González, 2006). Los resultados experimentales obtenidos en este trabajo fueron complementados con los resultados encontrados por González \& Leguizamón (2012).

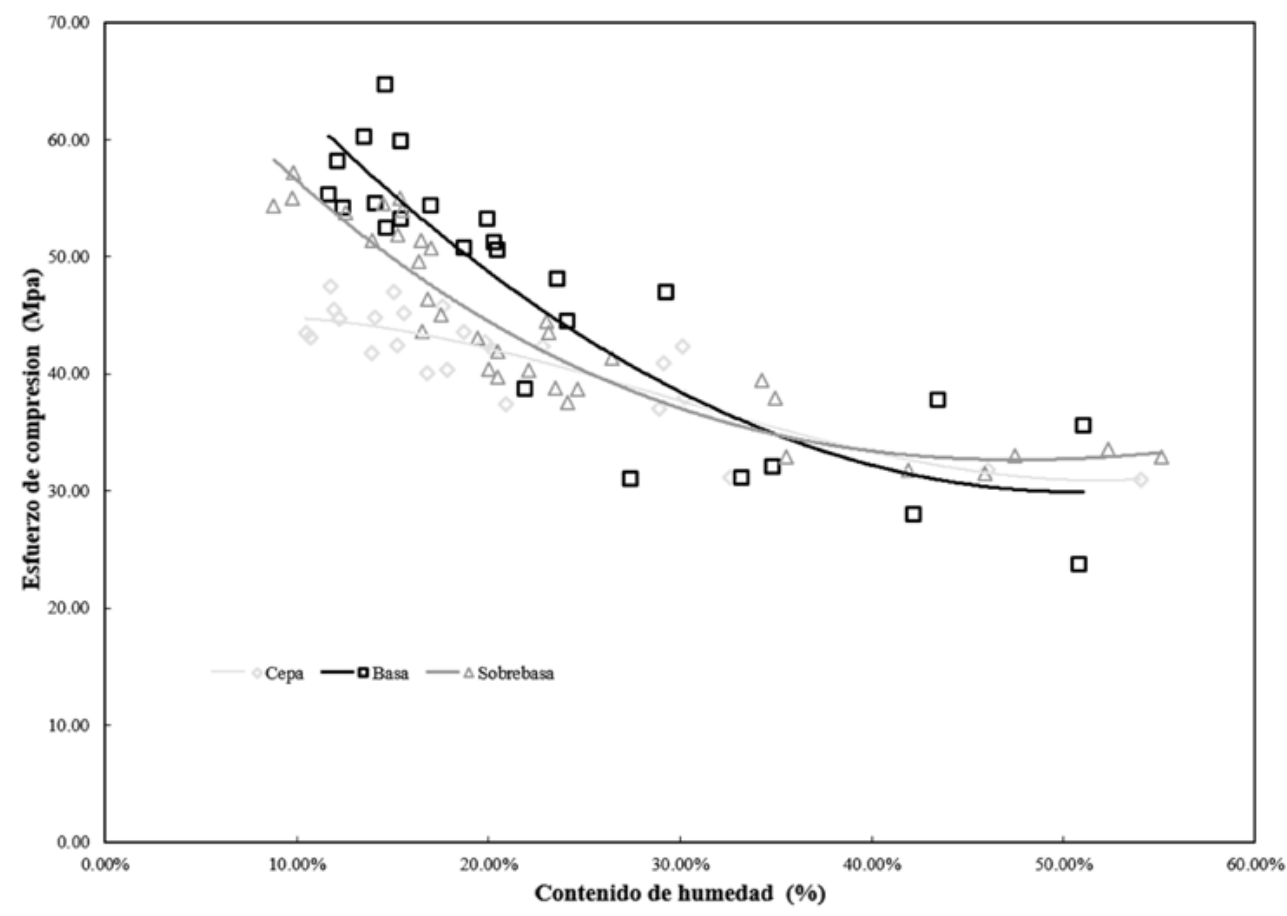

Figura 3. Variación de la resistencia a la compresión paralela en función del contenido de humedad. 
De acuerdo con lo que se observa en la figura 4, se evidencia que la basa y sobrebasa resisten mayores esfuerzos de compresión paralela en comparación con la cepa, la cual resiste un menor esfuerzo debido a que esta parte presenta una menor densidad de fibras (Liese, 1998), tal como lo han reportado diversos autores (Tan et al., 2011; Luna et al, 2012). Igualmente, se observa que en la cepa la pendiente de la figura esfuerzo último a compresión versus humedad es menos pronunciada en comparación con las zonas de basa y sobrebasa.

En la figura 4 se presenta la curva de esfuerzo a la compresión paralela en función del contenido de humedad para todas las partes de la guadua en altura. De esta gráfica se determinó que, de acuerdo con los ensayos de resistencia, el PSF de la $G$. angustifolia Kunth corresponde a $34.00 \%$, ya que a partir de ese $\mathrm{CH}$ presenta un cambio de la pendiente y la resistencia a la compresión se mantiene prácticamente constante.

Con estos resultados también fue posible plantear los factores de modificación de la resistencia a la compresión en la G. angustifolia, al encontrar que la resistencia a la compresión paralela a la fibra varía significativamente para contenidos de humedad entre el $10 \%$ y el $34 \%$, punto que se ha escogido como el PSF. En ese rango, la guadua puede disminuir su resistencia hasta en un $35 \%$ tal y como se muestra en la figura 4 . Utilizando la ecuación de la línea recta con los datos normalizados de la resistencia a la compresión entre $10 \%$ y $34 \%$ de $\mathrm{CH}$, se puede plantear un factor de modificación de la resistencia como se muestra en la tabla 1.

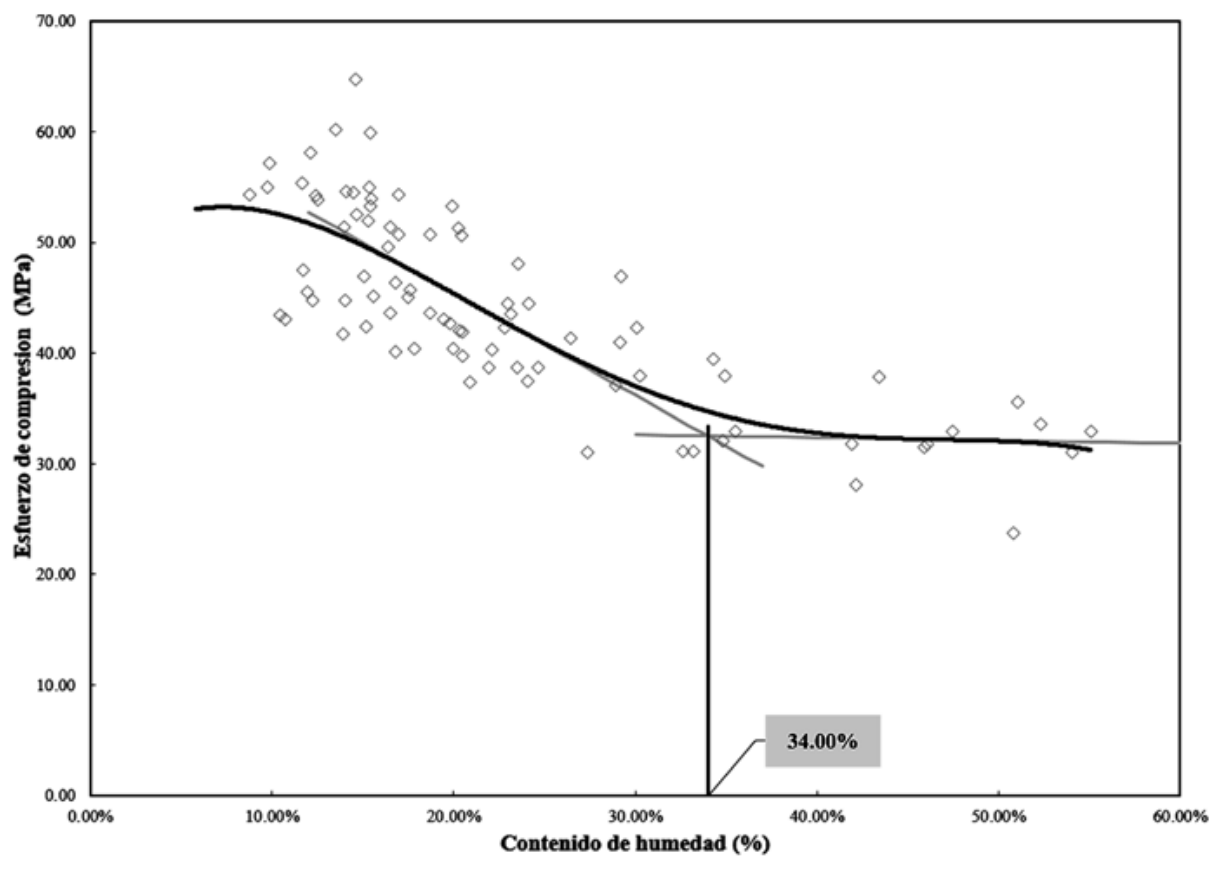

Figura 4. Variación de la resistencia a la compresión paralela a partir del contenido de humedad.

Tabla 1. Factores de modificación de la resistencia a la compresión paralela $\left(\boldsymbol{F}_{\mathrm{C}}\right)$ para diferentes contenidos de humedad por debajo del PSF.

\begin{tabular}{ccccccccccccc}
\hline $\mathbf{C H}(\boldsymbol{\%})$ & $\leq \mathbf{1 0} \%$ & $\mathbf{1 2} \%$ & $\mathbf{1 4} \%$ & $\mathbf{1 6} \%$ & $\mathbf{1 8} \%$ & $\mathbf{2 0} \%$ & $\mathbf{2 2} \%$ & $\mathbf{2 4} \%$ & $\mathbf{2 6} \%$ & $\mathbf{2 8} \%$ & $\mathbf{3 0} \%$ & $\geq \mathbf{3 4} \%$ \\
\hline $\mathrm{FM}\left(\boldsymbol{f}_{C}\right)$ & 1.00 & 0.98 & 0.95 & 0.92 & 0.89 & 0.86 & 0.83 & 0.80 & 0.77 & 0.74 & 0.71 & 0.65 \\
\hline
\end{tabular}




\section{DISCUSIÓN}

Los resultados presentados demuestran que el PSF de la G. angustifolia se encuentra alrededor del $34 \%$ de contenido de humedad. Este resultado es similar a lo presentado por Montoya (2007), Xu et al, (2014) y Jiang et al. (2015), quienes han establecido que otras especies de bambúes han presentado PSF alrededor del $30 \%$, lo cual han descubierto a partir de la variación de la resistencia mecánica ante diferentes solicitaciones de carga, o partir de las curvas de sorción del material. Sin embargo, otros autores como Hidalgo $(1981,2003)$ y Hamdan et al. (2007) también han planteado que el PSF en algunos bambúes es menor y puede estar alrededor del $24 \%$. En algunas investigaciones, el PSF han sido obtenido a través de isotermas de sorción, al exponer probetas de bambú en un ambiente completamente saturado, es decir, con una humedad relativa del $100 \%$ a diferentes temperaturas. Esta condición es muy diferente a la de tener probetas totalmente sumergidas, expuestas en ambientes saturados de agua en forma líquida, y no en forma de vapor, lo cual obviamente conduce a resultados diferentes. En otras investigaciones realizadas por el autor, se ha podido encontrar que la $\mathrm{G}$. angustifolia alcanza un $\mathrm{CH}$ de hasta el $26 \%$, en condiciones ambientales con humedades relativas por encima del 90 \% (Gutiérrez \& Briceño, 2015); sin embargo, esto no permite determinar que ese valor corresponde al del PSF.

La metodología propuesta permitió determinar que el bambú, al igual que la madera, no presenta variaciones dimensionales significativas en la dirección longitudinal debido a que la expansión lineal encontrada para valores de $\mathrm{CH}$ por encima del PSF no fue mayor a $0.8 \%$. Sin embargo, la expansión radial y tangencial sí puede ser de hasta un $6 \%$, lo cual coincide con lo encontrado para la madera (Forest Products Laboratory, 2010). Se puede confirmar que la $G$. angustifolia se contrae o expande principalmente en dirección radial, mientras que dirección axial presenta deformaciones mínimas debido al cambio en el $\mathrm{CH}$ (Liesse \& Köhl, 2015).
Se encontró que la parte de la guadua en altura, y la presencia o ausencia de nudo no son variables determinantes para establecer el PSF del material, ya que los valores de PSF no variaron en más de $1 \%$ con respecto al $\mathrm{CH}$ encontrado para cada variable de manera independiente. Igualmente, por las condiciones en las que se utiliza la guadua como material estructural, siempre es necesario contar con elementos que poseen tramos con o sin nudo, o con longitudes que abarcan diferentes partes en altura, por lo que siempre se deberá trabajar con un único valor de PSF sin importar estas variables geométricas. De acuerdo con Liesse \& Köhl (2015), cuando el contenido de humedad es relativamente bajo (por debajo del PSF), la expansión debida a la absorción de agua es prácticamente igual a la contracción debida a la adsorción, razón por la que los resultados presentados pueden utilizarse para describir fenómenos de contracción o expansión para $\mathrm{CH}$ por debajo del PSF. Según los resultados obtenidos, la dimensión radial alcanzó expansiones máximas de hasta un $6.5 \%$, las expansiones tangenciales máximas fueron de $5.1 \%$ y las longitudinales de máximo $0.8 \%$. Se puede observar que los cambios dimensionales en la dirección tangencial y radial del bambú son prácticamente iguales. Mientras la contracción tangencial de la madera es casi el doble de la contracción en la dirección radial. La contracción o expansión aparente del bambú en dirección radial es levemente mayor, aunque muy parecida a la contracción o expansión en dirección tangencial. Según Liesse \& Köhl (2015), esto puede atribuirse a dos efectos: en primer lugar, el bambú no contiene células orientadas radialmente como sí se presenta en la madera, lo que podría limitar la contracción en esa dirección; y, en segundo lugar, cuando los canutos de bambú se secan o se humedecen, el transporte principal de agua está dirigido radialmente a través de la lacuna y, por lo tanto, la inestabilidad dimensional ocurre principalmente en la dirección radial (Liesse \& Köhl, 2015).

Respecto a la variación de la resistencia a la compresión en función del contenido de humedad, los resultados obtenidos se asimilan a lo 
presentado actualmente en el reglamento colombiano de construcción resistente (AIS, 2010), el cual determina que la resistencia puede disminuirse hasta en un $30 \%$, tal como lo plantea Jiang et al. (2012), Xu et al. (2014) y Wang et al. (2015). Otros autores, a pesar de coincidir en la disminución en la resistencia, han planteado que esta disminución se da entre un 12 y un $18 \%$ de $\mathrm{CH}$, sugiriendo que el PSF es igual a $19 \%$ (AIS, 2010; Kaminski et al., 2016b). Sin embargo, esto difiere con lo encontrado en esta investigación. Es importante mencionar que muchas de estas investigaciones se han realizado con diferentes especies de bambú, y aunque también han demostrado que la disminución de la resistencia a la compresión paralela puede llegar a ser de hasta un $30 \%$, hasta el momento es muy poco lo que se ha publicado para la especie $G$. angustifolia Kunth.

\section{CONCLUSIONES}

La dimensiones radiales y transversales presentaron una mayor expansión lineal para todas las partes de la guadua en comparación con la dimensión longitudinal, ya que en general la dimensión radial alcanzó expansiones máximas de hasta un $6.5 \%$, las dimensiones tangenciales de $5.1 \%$ y las longitudinales de máximo $0.8 \%$.

Se encontró una ecuación matemática que permite describir la expansión lineal porcentual que experimentaría un elemento de guadua rolliza en su dirección radial, lo cual podría ser de gran interés para las diferentes industrias que trabajan con el material.

A partir de los ensayos realizados donde se midieron los cambios dimensionales, así como a partir de los ensayos de resistencia a compresión paralela, se determinó que el PSF para la guadua se encuentra alrededor de $34 \%$ de $\mathrm{CH}$.

Se plantearon los factores de modificación de la resistencia a la compresión paralela a partir de la variación en el contenido de humedad, encontrando que la resistencia de la guadua puede disminuirse hasta en un $35 \%$ para contenidos de humedad comprendidos entre el $10 \%$ y el $34 \%$.

Los resultados encontrados permitirán a profesionales, diseñadores estructurales y constructores con bambú guadua comprender el rango de contenido de humedad en el cual el material presenta cambios en sus dimensiones, así como variación de sus propiedades mecánicas.

\section{AGRADECIMIENTOS}

Los autores agradecen a la Universidad La Gran Colombia, por el apoyo y la financiación en el desarrollo y presentación de los resultados de esta investigación. Igualmente, se agradece a cada uno de los docentes que aportaron sus conocimientos para la realización de este proyecto.

\section{CONFLICTO DE INTERESES}

Los autores declaran no tener conflicto de intereses.

\section{CONTRIBUCIÓN POR AUTOR}

El autor único es responsable de la obra en todos los aspectos que condujeron a la elaboración de su publicación.

\section{REFERENCIAS BIBLIOGRÁFICAS}

\section{Asociación Colombiana de ingenieria Sísmica (AIS).} (2010). Reglamento Colombiano de Construcción Sismo Resistente NSR-10 Titulo G-Estructuras de Madera y Estructuras de Guadua. Bogotá: AIS. 168 p.

Junta del Acuerdo de Cartagena. (1984). Manual de diseño para maderas del grupo Andino. Lima: Junta del Acuerdo de Cartagena. 560 p.

Correal, J.F. (2016). Bamboo design and construction. En K. Harries \& B. Sharma (eds.), Nonconventional 
and Vernacular Construction Materials (pp. 393431). Duxford: Elsevier Ltd.

Dowling, D.M. (2004). Adobe housing in El Salvador: Earthquake performance and seismic improvement. In W.I. Rose, J.J. Bommer, D.L. López, M.J. Carr, \& J.J. Major (eds.), Natural Hazards in El Salvador: Geological Society of America. DOI: https:// doi.org/10.1130/0-8137-2375-2.281

Gatóo, A., Sharma, B., Bock, M., Mulligan, H., \& Ramage, M.H. (2014). Sustainable structures: bamboo standards and building codes. Proceedings of the Institution of Civil Engineers-Engineering Sustainability, 167(5), 189-196. DOI: https://doi. org/10.1680/ensu.14.00009

Gonzalez, J.C., \& Leguizamon, Y. (2012). Determinación de la resistencia a compresión paralela a la fibra de la Guadua (angustifolia kunth) en función del contenido de humedad. (Trabajo de grado, Ingeniero Civil). Bogotá: Universidad la Gran Colombia. $133 \mathrm{p}$.

Gonzalez, E., \& Takeuchi, C. (2007). Resistencia a la compresión paralela a la fibra de la Guadua angustifolia y determinación del módulo de elasticidad. Ingeniería y Universidad, Enero-Junio, 89-103.

Gonzalez, E., \& Diaz, J.F. (1992). Propiedades físicas y mecánicas de la guadua (Guadua angustifolia Kunth). Medellin: Universidad Nacional de Colombia.

Grosser, D., \& Liese, W. (1971). On the anatomy of Asian bamboo with especial reference to their vascular bundles. Wood Science and Technology, 5, 22. DOI: https://doi.org/10.1007/BF00365061

Gutiérrez, M. (2011). Factor de corrección por contenido de humedad para la resistencia a tensión paralela a la fibra de la Guadua angustifolia Kunth. (Trabajo de grado, Maestría en Ingienería). Bogota: Universidad Nacional de Colombia. 151 p.

Gutierrez, M., \& Takeuchi, C. (2014). Efecto del contenido de humedad en la resistencia a tensión paralela a la fibra del bambú Guadua angustifolia Kunth. Scientia et Technica, 19(3), 6.

Forest Products Laboratory. (2010). Wood handbook-Wood as an engineering material. General Technical Report FPL-GTR-190. Madison, WI, EE.
UU.: Department of Agriculture, Forest Service, Forest Products Laboratory. 508 p.

Gutiérrez, M., \& Briceño, P.A. (2015). Equilibrium Moisture Content and Sorption Isotherms determination for different conditions of relative humidity and temperature in the bamboo Guadua angustifolia Kunth. Artículo presentado en 16th NOCMAT 2015, Winnipeg, Canadá.

Hamdan, H., Hill, A.Z., U.M.K., A., \& Latif, M.A. (2007). Equilibrium moisture content and volumetric changes of Gigantochloa Scortechinii. Journal of Tropical Forest Science, 19(1), 7.

Hidalgo, O. (1981). Manual de construccion con bambu. Bogotá: Universidad Nacional de Colombia, Cibam, Facultad de Artes. 87 p.

Hidalgo, O. (2003). Bamboo: The Gift of the Gods. Bogotá: Óscar Hidalgo-López. 553 p.

Instituto Colombiano de Normas Técnicas y Certificación (Icontec). (2007a). Métodos de ensayo para determinar las propiedades físicas y mecánicas de la Guadua angustifolia Kunth (NTC5525). Bogotá: Icontec. 22 p.

Icontec. (2007b). Norma Técnica Colombiana 5301. Preservación y secado del culmo de Guadua angustifolia Kunth. Bogotá: Icontec. 5 p.

Jiang, Z., Wang, H., Tian, G., Liu, X., \& Yu, Y. (2012). Sensitivity of several selected mechanical properties of moso bamboo to moisture content change under the fibre saturation point. BioResources, 7(4), 5048-5058. DOI: https://doi.org/10.15376/ biores.7.4.5048-5058

Jiang, Z., Wang, H., Tian, G., Liu, X., \& Yu, Y. (2015). Sensitivity of several selected mechanical properties of Moso Bamboo to moisture content change under fibre saturation point. BioResources, 7(4), 10.

Kaminski, S., Lawrence, A., \& Trujillo, D. (2016). Structural use of bamboo. Part 1: Introduction to bamboo. The Structural Engineer. (pp. 40-43).

Kaminski, S., Lawrence, A., Trujillo, D., Feltham, I., \& Lopez, L. F. (2016). Structural use of bamboo. Part 3: Design values. The Structural Engineer. (pp. 42-45).

Liese, W. (1998). The Anatomy of Bamboo Culms. Inbar Technical Report N 18. Beijing: Inbar. 208 p. 
Liese, W., \& Köhl, M. (2015). Bamboo: The Plant and its Uses (Tropical Forestry). Cham: Springer International Publishing. 356 p. DOI: https://doi. org/10.1007/978-3-319-14133-6

Luna, P., Lozano, J., Takeuchi, C., \& Gutierrez Gonzalez, M. (2012) Experimental determination of allowable stresses for Bamboo Guadua angustifolia Kunth structures. Key Engineering Materials, 517, 76-80. DOI: https://doi.org/10.4028/www.scientific.net/KEM.517.76

Luna, P., Takeuchi, C., Granados, G., Lamus, F., \& Lozano, J. (2011). Metodología de diseño de estructuras en guadua angustifolia como material estructural por el método de esfuerzos admisibles. Revista Educación en Ingeniería, 6(11), 66-75. DOI: http:// dx.doi.org/10.26507/rei.v6n11.117

Montoya, J.A. (2007). Determinación de las curvas isotermas de sorción y el PSF -Punto de saturación de fibra de la especie de bambú Phyllostachys pubescens Mazel. Scientia et Technica, 35.

Sharma, S.N. (1988) Seasoning behavior and related properties of some Indian species of bamboo. Indian For, 114(10), 613-621.

Tan, T., Rahbar, N., Allameh, S. M., Kwofie, S., Dissmore, D., Ghavami, K., \& Soboyejo, W.O. (2011). Mechanical properties of functionally graded hierarchical bamboo structures. Acta Biomater, 7(10), 3796-3803. DOI: https://doi.org/10.1016/j. actbio.2011.06.008

Wang, H., Wang H., Wanju L., Ren D., \& Yu Y. (2013). Effects of moisture content on the mechanical properties of moso bamboo at the macroscopic and ceIlular levels. Bioresources, 8(4), 5475-5484. DOI: https://doi.org/10.15376/biores.8.4.5475-5484

Wang, H., Tian, G., Li, W., Ren, D., Zhang, X., \& Yu, Y. (2015). Sensitivity of bamboo fiber longitudinal tensile properties to moisture content variation under the fiber saturation point. Journal of Wood Science, 61(3), 262-269. DOI: https://doi.org/10.1007/ s10086-015-1466-y

Xu, Q., Harries, K., Li, X., Liu, Q., \& Gottron, J. (2014). Mechanical properties of structural bamboo following immersion in water. Engineering Structures, 81, 230-239. DOI: https://doi.org/10.1016/j. engstruct.2014.09.044

Zea, E., \& Habert, G. (2015). Regionalizing the Environmental Impact of Bamboo-Based Buildings by Integrating Life Cycle Assessment with Geographic Information Systems. A Comparative Case-Study in Colombia. Articulo presentado en 10th World Bamboo Congres, Damyang: Corea del Sur. 